\title{
Connected Weak Edge Detour Number of a Graph
}

\author{
J. M. Prabakar*and S. Athisayanathan ${ }^{\dagger}$
}

\begin{abstract}
Certain general properties of the detour distance, weak edge detour set, connected weak edge detour set, connected weak edge detour number and connected weak edge detour basis of graphs are studied in this paper. Their relationship with the detour diameter is discussed. It is proved that for each pair of integers $k$ and $n$ with $2 \leq k \leq n$, there exists a connected graph $G$ of order $n$ with $c d n_{w}(G)=k$. It is also proved that for any three positive integers $R, D, k$ such that $k \geq D$ and $R<D \leq 2 R$, there exists a connected graph $G$ with $\operatorname{rad}_{D} G$ $=R, \operatorname{diam}_{D} G=D$ and $c d n_{w}(G)=k$.
\end{abstract}

Keywords: Detour, Detour number, Weak edge detour number, Connected weak edge detour number

Mathematics Subject Classification (2010): 05 C12

\section{Introduction}

Graphs are discrete structures that represent objects and their relations among them. For a graph $G=(V, E)$, with the vertex (object) set $V$ and edge set, i.e., the set of relations, $E$, the order and size of $G$ are denoted by $n$ and $m$ respectively. For basic definitions and terminologies we refer to $[4,1]$. Throughout this paper $G$ denotes a finite undirected connected simple graph with at least two vertices.

For vertices $u$ and $v$ in $G$, the distance $d(u, v)$ is the length of a shortest $u-v$ path in $G$. A $u-v$ path of length $d(u, v)$ is called a $u-v$ geodesic. For a vertex $v$ of $G$, the eccentricity $e(v)$ is the distance between $v$ and a vertex farthest from $v$. The minimum eccentricity among the vertices

${ }^{*}$ St. Xavier's College (Autonomous), Palayamkottai 627 002; jmpsxc@gmail.com †St. Xavier’s College; athisxc@gmail.com

Received: April 2016. Reviewed: May 2016 
of $G$ is the radius, $\mathrm{rad} G$ and the maximum eccentricity is its diameter, $\operatorname{diam} G$ of $G$.

The detour distance $D(u, v)$ is the length of a longest $u-v$ path in $G$ for vertices $u$ and $v$ in $G$. A $u-v$ path of length $D(u, v)$ is called a $u-v$ detour. For a vertex $v$ of $G$, the detour eccentricity $e_{D}(v)$ is the detour distance between $v$ and a vertex farthest from $v$. The detour radius, $\operatorname{rad}_{D} G$ of $G$ is the minimum detour eccentricity among the vertices of $G$, while the detour diameter, $\operatorname{diam}_{D} G$ of $G$ is the maximum detour eccentricity among the vertices of $G$. These concepts were studied by Chartrand et al. [2]

A vertex $x$ is said to lie on a $u-v$ detour $P$ if $x$ is a vertex of $P$ including the vertices $u$ and $v$. A set $S \subseteq V$ is called a detour set if every vertex $v$ in $G$ lies on a detour joining a pair of vertices of $S$. The detour number $\operatorname{dn}(G)$ of $G$ is the minimum order of a detour sets and any detour set of order $\operatorname{dn}(G)$ is called a detour basis of $G$. A vertex $v$ that belongs to every detour basis of $G$ is a detour vertex in $G$. If $G$ has a unique detour basis $S$, then every vertex in $S$ is a detour vertex in $G$.[3]

A set $S \subseteq V$ is called a weak edge detour set of $G$ if every edge in $G$ has both its ends in $S$ or it lies on a detour joining a pair of vertices of $S$. The weak edge detour number $d n_{w}(G)$ of $G$ is the minimum order of its weak edge detour sets and any weak edge set of order $d n_{w}(G)$ is called a weak edge detour basis of $G$. These concepts were studied by Santhakumaran and Athisayanathan. [5]

A set $S \subseteq V$ is called a connected detour set of $G$ if $S$ is a detour set of $G$ and the subgraph $G\langle S\rangle$ induced by $S$ is connected. The connected detour number $c d n(G)$ of $G$ is the minimum order of its connected detour sets and any connected detour set of order $c d n(G)$ is called connected detour basis of G.[6] This motivated us to introduce and investigate the concepts of connected weak edge detour set and connected weak edge detour number of a graph $G$.

The following theorems are used in this paper for proving the results.

Theorem 1.1. [3] Every end-vertex of a non-trivial connected graph $G$ belongs to every detour set of $G$. Also if the set $S$ of all end-vertices of $G$ is a detour set, then $S$ is the unique detour basis for $G$.

Theorem 1.2. [5] Every end-vertex of a non-trivial connected graph $G$ belongs to every weak edge detour set of $G$. Also if the set $S$ of all endvertices of $G$ is a weak edge detour set, then $S$ is the unique weak edge detour basis for $G$.

Theorem 1.3. [5] If $T$ is a non-trivial tree with $k$ end-vertices, then $d n(T)=d n_{w}(T)=k$. 


\section{Connected Weak Edge Detour Number of a Graph}

Definition 2.1. Let $G=(V, E)$ be a connected graph with at least two vertices. A set $S \subseteq V$ is a connected weak edge detour set of $G$ if $S$ is a weak edge detour set of $G$ and the subgraph $\langle S\rangle$ induced by $S$ is connected. The connected weak edge detour number $c d n_{w}(G)$ of $G$ is the minimum order of its connected weak edge detour sets and any connected weak edge detour set of order $c d n_{w}(G)$ is called a connected weak edge detour basis of $G$.

Example 2.2. For the graph $G$ given in Figure 2.1, it is clear that no two element subset of $V$ is a connected weak edge detour set of $G$.The set $S=\left\{v_{1}, v_{2}, v_{3}\right\}$ is a connected weak edge detour basis of $G$ so that $c d n_{w}(G)=3$. The set $S_{1}=\left\{v_{1}, v_{2}, v_{4}\right\}$ and $S_{2}=\left\{v_{1}, v_{3}, v_{5}\right\}$ are also connected weak edge detour bases of $G$. Thus there can be more than one connected weak edge detour basis for a graph $G$.

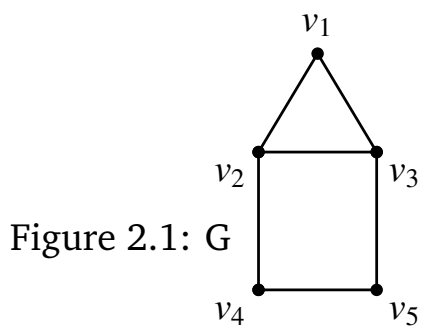

Remark 2.3. Every connected weak edge detour set is a weak edge detour set but the converse is not true. For the graph $G$ given in figure 2.1 , the set $U=\left\{v_{1}, v_{4}, v_{5}\right\}$ is a weak edge detour set but not a connected weak edge detour set of $G$.

Example 2.4. For the graph $G$ given in Figure 2.2, the set $S_{1}=\left\{v_{2}, v_{3}\right\}$ is a connected weak edge detour basis for $G$ so that $c d n_{w}(G)=d n_{w}(G)=2$.

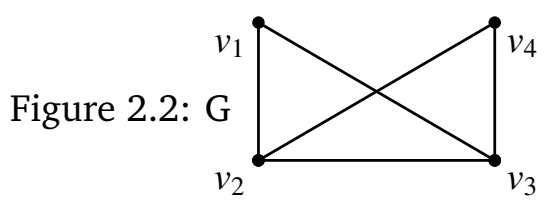

Theorem 2.5. For any graph $G$ of order $n \geq 2,2 \leq c d n_{w}(G) \leq n$.

Proof. A connected weak edge detour set needs at least two vertices so that $c d n_{w}(G) \geq 2$ and the set of all vertices of $G$ is a connected weak edge detour set of $G$ so that $c d n_{w}(G) \leq n$. Thus $2 \leq c d n_{w}(G) \leq n$.

Remark 2.6. The bounds in Theorem 2.5 are sharp. For the complete graph $K_{2}, c d n_{w}\left(K_{2}\right)=2$. The set of all vertices of path $P_{n}(n \geq 2)$ is 
its unique connected weak edge detour set so that $c d n_{w}(G)=n$. Also the inequalities in Theorem 2.5 can be strict. For the graph $G$ given in Figure 2.1, $n=5, c d n_{w}(G)=3$ so that $2<c d n_{w}(G)<n$. Thus the complete graph $K_{2}$ has the smallest possible connected weak edge detour number 2 and the non-trivial paths have the largest possible connected weak edge detour number $n$.

Definition 2.7. A vertex $v$ in a graph $G$ is a connected weak edge detour vertex if $v$ belongs to every connected weak edge detour basis of $G$. If $G$ has a unique connected weak edge detour basis $S$, then every vertex in $S$ is a connected weak edge detour vertex of $G$.

Example 2.8. For the graph $G$ given in Figure 2.3, $S=\{u, v, w, x, y\}$ is the unique connected weak edge detour basis so that every vertex of $S$ is a connected weak edge detour vertex of $G$.

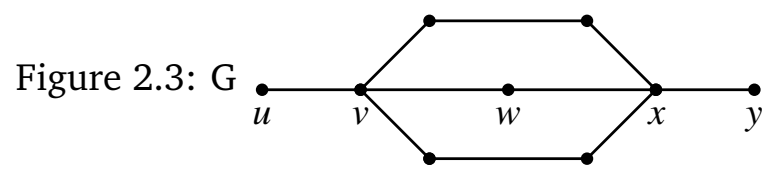

Example 2.9. For the graph $G$ given Figure 2.4, $S_{1}=\{u, s, w, t, v\}$, $S_{2}=\{u, s, x, t, v\}$ and $S_{3}=\{u, s, y, t, v\}$ are the connected weak edge detour bases of $G$ so that $u, s, t$ and $v$ are the connected weak edge detour vertices of $G$.

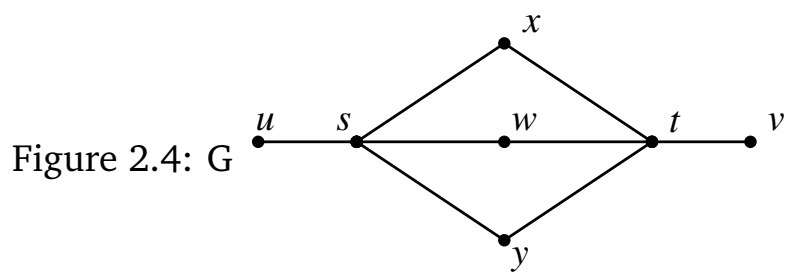

In the following theorems we show that there are certain vertices in a non-trival connected graph $G$ that are connected weak edge detour vertices of $G$.

Theorem 2.10. Every end-vertex of a non-trivial connected graph $G$ belongs to every connected weak edge detour set of $G$.

Proof. Let $v$ be an end-vertex of $G$ and $u v$ an edge in $G$ incident with $v$. Then $u v$ is either an initial edge or the terminal edge of any detour containing the edge $u v$. Hence it follows that $v$ belongs to every connected weak edge detour set of $G$.

Theorem 2.11. Let $G$ be a connected graph with cut-vertices and $S$ a connected weak edge detour set of $G$. Then for any cut-vertex $v$ of $G$, every component of $G-v$ contains an element of $S$. 
Proof. Let $v$ be a cut-vertex of $G$ such that one of the components, say $C$ of $G-v$ contains no vertex of $S$. Then by Theorem 2.10, $C$ does not contain any end-vertex of $G$. Hence $C$ contains at least one edge, say $u w$. Since $S$ is a connected weak edge detour set there exists vertices $x, y \in S$ such that $u w$ lies on some $x-y$ detour $P: x=$ $u_{0}, u_{1}, \ldots, u, w, \ldots u_{t}=y$ in $G$ or both the ends $u$ and $w$ of the edge $u w$ are in $S$. Suppose that $u w$ lies on the detour $P$. Let $P_{1}$ be the $x-u$ subpath of $P$ and $P_{2}$ be the $u-y$ subpath of $P$. Since $v$ is a cut-vertex of $G$ both $P_{1}$ and $P_{2}$ contain $v$ so that $P$ is not a detour, which is a contradiction. Suppose that $u$ and $w$ are in $S$, then $C$ contains vertices of $S$, which is again a contradiction.

Theorem 2.12. Let $G$ be a connected graph with cut-vertices. Then every cut-vertex of $G$ belongs to every connected weak edge detour set of $G$.

Proof. Let $G$ be a connected graph and $v$ be a cut-vertex of $G$. Let $G_{1}$, $G_{2}, \ldots, G_{k}(k \geq 2)$ be the components of $G-v$. Let $S$ be any connected weak edge detour set of $G$. Then by Theorem 2.11, $S$ contains at least one element from each component $G_{i}(1 \leq i \leq k)$ of $G-v$. Since $\langle S\rangle$ is connected it follows that $v \in S$.

Corollary 2.13. All the end-vertices and the cut-vertices of a connected graph $G$ belong to every connected weak edge detour set of $G$.

Proof. Proof is immediate from the Theorems 2.10 and 2.12.

Remark 2.14. For the graph $G$ given in Figure 2.5, $S_{1}=\{u, w, x\}, S_{2}=$ $\{u, w, y\}, S_{3}=\{v, w, x\}$ and $S_{4}=\{v, w, y\}$ are the four connected weak edge detour bases. The cut vertex $w$ belongs to every connected weak edge detour basis so that the cut-vertex $w$ is the unique connected weak edge detour vertex of $G$.

Figure 2.5: G

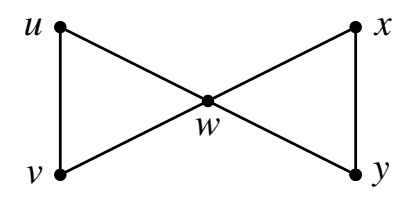

Corollary 2.15. If $T$ is a tree of order $n \geq 2$, then $c d n_{w}(T)=n$.

Proof. Corollary 2.13 gives the proof.

Corollary 2.16. For any connected graph $G$ with $k$ end-vertices and $l$ cut-vertices, $\max \{2, k+l\} \leq c d n_{w}(G) \leq n$.

Proof. The Theorem 2.5 and the corollary 2.13 give the proof. 
For the graph $H$ and an integer $k \geq 1$, we write $k H$ for the union of the $k$ disjoint copies of $H$.

Theorem 2.17. Let $G=\left(K_{n_{1}} \cup K_{n_{2}} \cup \ldots \cup K_{n_{r}} \cup k K_{1}\right)+v$ be a block graph of order $n \geq 4$ such that $r \geq 1$, each $n_{i} \geq 2$ and $n_{1}+n_{2}+\ldots+n_{r}+k=n-1$. Then $c d n_{w}(G)=r+k+1$.

Proof. Let $u_{1}, u_{2}, \ldots u_{k}$ be the end-vertices of $G$. Let $S$ be any connected weak edge detour set of $G$. Then by Corollary 2.13, $v \in S$ and $u_{i} \in S(1 \leq i \leq k)$. Also by Theorem 2.11, $S$ contains a vertex from each component $K_{n_{i}}(1 \leq i \leq r)$. Now choose exactly one vertex $v_{i}$ from each $K_{n_{i}}$ such that $v_{i} \in S$. Then $|S| \geq r+k+1$. Let $T=\left\{v, v_{1}, v_{2}, \ldots v_{r}, u_{1}, u_{2}, \ldots, u_{k}\right\}$. Since every edge in $G$ has both its ends in $T$ or it lies on a detour joining a pair of vertices of $T$, it follows that $T$ is a weak edge detour basis of $G$. Also, since $\langle T\rangle$ is connected, $c d n_{w}(G)=r+k+1$.

Remark 2.18. If the blocks of the graph $G$ in Theorem 2.17 are not complete, then the theorem is not true. For the graph $G$ given in Figure 2.6 there are two blocks and $\left\{v_{4}, v_{9}, v_{5}, v_{7}\right\}$ is a connected weak edge detour basis so that $c d n_{w}(G)=4$.

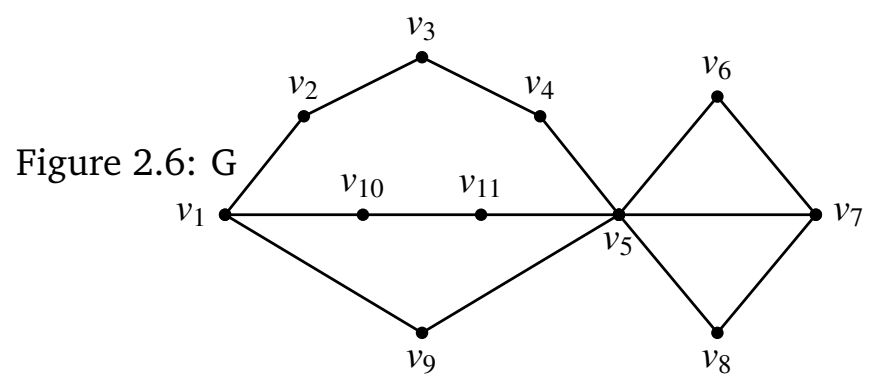

Theorem 2.19. Let $G$ be the complete graph $K_{n}(n \geq 2)$. Then a set $S \subseteq V$ is a connected weak edge detour basis of $G$ if and only if $S$ consists of any two vertices of $G$.

Proof. Let $G$ be the complete graph $K_{n}(n \geq 2)$ and $S=\{u, v\}$ be any set of two vertices of $G$. It is clear that $D(u, v)=n-1$. Let $x y \in E$. If $x y=u v$, then both its ends are in $S$. Let $x y \neq u v$. If $x \neq u$ and $y \neq v$, then the edge $x y$ lies on the $u-v$ detour $P: u, x, y, \ldots, v$ of length $n-1$. If $x=u$ and $y \neq v$, then the edge $x y$ lies on the $u-v$ detour $P: u=x, y, \ldots, v$ of length $n-1$. Hence $S$ is a connected weak edge detour of $G$. Since $|S|=2, S$ is a connected weak edge detour basis of $G$.

Conversely, let $S$ be a connected weak edge detour basis of $G$. Let $S^{\prime}$ be any set consisting of two vertices of $G$. Then as in the first part of this theorem $S^{\prime}$ is a connected weak edge detour basis of $G$. Hence $|S|=\left|S^{\prime}\right|=2$ and it follows that $S$ consists of any two vertices of $G$. 
Theorem 2.20. Let $G$ be a cycle of order $n \geq 3$. Then a set $S \subseteq V$ is a connected weak edge detour basis of $G$ if and only if $S$ consists of any two adjacent vertices of $G$.

Proof. Let $S=\{u, v\}$ be any set of two adjacent vertices of $G$. It is clear that $D(u, v)=n-1$. Then every edge $e \neq u v$ of $G$ lies on the $u-v$ detour and the both ends of the edge $u v$ belong to $S$ so that $S$ is a connected weak edge detour set of $G$. Since $|S|=2, S$ is a connected weak edge detour basis of $G$.

Conversely, assmume that $S$ is a connected weak edge detour basis of $G$. Let $S^{\prime}$ be any set of two adjacent vertices of $G$. Then as in the first part of this theorem $S^{\prime}$ is a connected weak edge detour basis of $G$. Hence $|S|=\left|S^{\prime}\right|=2$. Let $S=\{u, v\} \subseteq V$. If $u$ and $v$ are not adjacent, it is clear that $u$ and $v$ are not connected. Thus $S$ consists of any two adjacent vertices of $G$.

Theorem 2.21. Let $G$ be the complete bipartite graph $K_{m, n}(2 \leq m \leq n)$. Then a set $S \subseteq V$ is a connected weak edge detour basis of $G$ if and only if $S$ consists of any two adjacent vertices of $G$.

Proof. Let $X$ and $Y$ be the bipartite sets of $G$ with $|X|=m$ and $|Y|=n$. Let $S=\{u, v\}$, where $u \in X$ and $v \in Y$ be any two adjacent vertices of $G$. It is clear that $D(u, v)=2 m-1$. Then every edge $e \neq u v$ of $G$ lies on the $u v$-detour and the both ends of the edge $u v$ belongs to $S$ so that $S$ is a connected weak edge detour set of $G$. Since $|S|=2, S$ is a connected weak edge detour basis of $G$.

Conversely, assume that $S$ is a connected weak edge detour basis of $G$. Let $S^{\prime}$ be any set of two adjacent vertices of $G$. Then as in the first part of this theorem $S^{\prime}$ is a connected weak edge detour basis of $G$. Hence $|S|=\left|S^{\prime}\right|=2$. Let $S=\{u, v\} \subseteq V$. If $u$ and $v \in X$ or $Y$ it is clear that $u$ and $v$ are not connected. Thus $S$ consists of any two adjacent vertices of $G$.

Corollary 2.22. (a) If $G$ is the complete graph $K_{n}$, then $c d n_{w}(G)=2$.

(b) If $G$ is the complete bipartite graph $K_{m, n}(2 \leq m \leq n)$, then $c d n_{w}(G)=2$.

(c) If $G$ is the cycle $C_{n}$, then $c d n_{w}(G)=2$.

Proof. (a) It follows from Theorem 2.19.

(b) It follows from Theorem 2.21.

(c) It follows from Theorem 2.10.

The following theorems give realization results. 
Theorem 2.23. For each pair of integer $k$ and $n$ with $2 \leq k \leq n$, there exists a connected graph $G$ of order $n$ with $c d n_{w}(G)=k$.

Proof. Case 1. $k=n$. Then any tree of order $n$ has the desired property by Corollary 2.15 .

Case 2. $2=k<n$, the cycle $C_{n}$ has the desired property by Corollary $2.22(c)$.

Case 3. $2<k<n$. Let $G$ be the graph obtained from the cycle $C_{n-k+2}: u_{1}, u_{2}, \ldots . u_{n-k+2}, u_{1}$ of order $n-k+2$ by adding $k-2$ new vertices $v_{1}, v_{2}, \ldots v_{k-2}$ and joining each vertex $v_{i}(1 \leq i \leq k-2)$ to $u_{1}$. The resulting graph $G$ is connected of order $n$ and is shown in Figure 2.7. Now we show that $c d n_{w}(G)=k$. Let $S=\left\{u_{1}, v_{1}, v_{2}, \ldots . v_{k-2}\right\}$ be the set of all end-vertices together with the cut-vertex $u_{1}$ of $G$. It is clear that $S$ is not a connected weak edge detour set of $G$. Let $T=S \cup\left\{u_{2}\right\}$. Then every edge of $G$ has both its ends in $T$ or it lies on a detour joining a pair of vertices of $T$ and also $<\mathrm{T}>$ is a connected so that $T$ is a connected weak edge detour basis of $G$, so that $c d n_{w}(G)=k$.

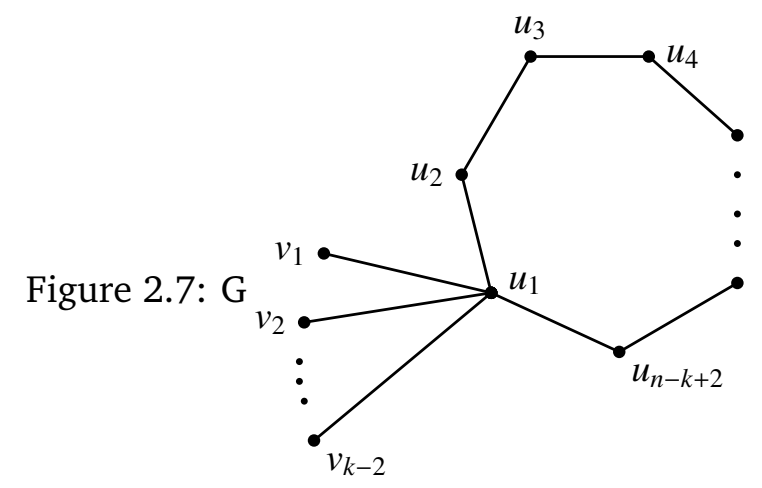

Theorem 2.24. For each positive integer $k \geq 2$ there exists a connected graph $G$ and a vertex $v$ of degree $k$ in $G$ such that $v$ belongs to a connected weak edge detour basis of $G$ and $c d n_{w}(G)=k$.

Proof. Case 1. $k=2$, the complete graph $K_{3}$ has a desired properties by Corollary $2.22(a)$.

Case 2. $k>2$, let $G$ be the graph obtained from the complete graph $K_{3}$, where $V\left(K_{3}\right)=\left\{v_{1}, v_{2}, v_{3}\right\}$ by adding $k-2$ new vertices $u_{1}, u_{2}, \ldots ., u_{k-2}$ and joining $u_{i}(1 \leq i \leq k-2)$ to $v_{1}$. The resulting graph $G$ is connected of order $n$ and is shown in the Figure 2.8. Then $\operatorname{deg}_{G} v_{1}=k$. Let $S=\left\{u_{1}, u_{2}, \ldots . . u_{k-2}, v_{1}\right\}$ be the set of all end-verties and cut-verties. However, by Corollary 2.13, $S$ is not a connected weak edge detour set of $G$. Let $T=S \cup\{v\}$, where $v \in\left\{v_{2}, v_{3}\right\}$ is a vertex in $K_{3}$. Then $T$ is a connected weak edge detour basis of $G$ and hence so that $c d n_{w}(G)=$ $k$. 
Figure 2.8: G

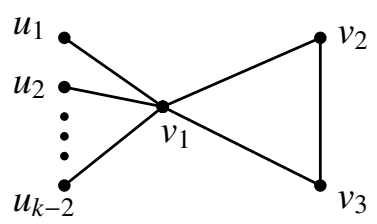

Theorem 2.25. For every pair of positive integer $a, b$ with $2 \leq a \leq b$, there exists a connected graph $G$ such that $d n_{w}(G)=a$ and $c d n_{w}(G)=b$.

Proof. Case 1: $a=b$, we have the following two sub cases.

Sub case (i): $a=2$, the complete graph $K_{2}$ has the desired property. Sub case (ii): $a>2$. Let $C_{3}: u_{1}, u_{2}, u_{3}$ be the cycle of length 3 . Now, by adding $a-2$ new vertices $v_{1}, v_{2}, \ldots \ldots v_{a-2}$ and joining the vertex $u_{2}$ as shown in the Figure 2.9. Let $S=\left\{v_{1}, v_{2}, \ldots \ldots v_{a-2}, u_{2}\right\}$ be the set of all end vertices and cut-verties of $G$. It is clear that $S$ is not a weak edge detour set of $G$. Let $T=S \cup\{u\}$, where $u \in\left\{u_{1}, u_{3}\right\}$ is a vertex in $C_{3}$. Then $T$ is a weak edge detour basis of $G$ so that $d n_{w}(G)=a$. Also the sub graph $\langle T\rangle$ induced by $T$ is connected so that $c d n_{w}(G)=a$.

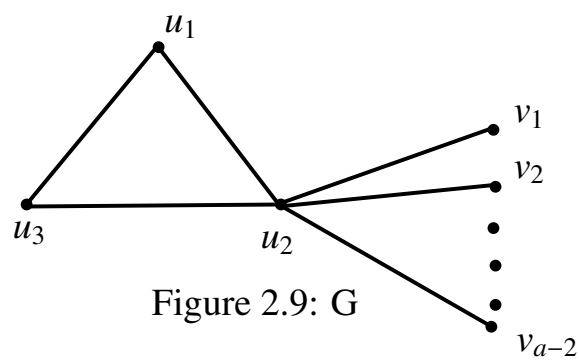

Case 2: $a<b$. Let $G$ be any tree with $a$ end -vertices and $b-a$ cut-vertices. Then by Theorem 1.3, $d n_{w}(G)=a$ and by Corollary 2.15, $c d n_{w}(G)=b$.

\section{Connected Weak Edge Detour Number and Detour Diameter of a graph}

In [3], an upper bound for the detour number, of a graph is given in terms of its order and detour diameter $D$ as follows:

Proposition $A$ [3] If $G$ is a non-trival connected graph of order $n \geq 3$ and detour diameter $D$, then $\operatorname{dn}(G) \leq n-D+1$.

Remark 3.1. In the case of weak edge detour number $d n_{w}(G)$ of a graph $G$ it is show in [5] that, there are graphs $G$ for which $d n_{w}(G)=n-D+1$, 
$d n_{w}(G)>n-D+1$ and $d n_{w}(G)<n-D+1$. Similarly, in the case of connected weak edge detour number $c d n_{w}(G)$ of the graph $G$, we show that there are graphs for which $c d n_{w}(G)=n-D+1, c d n_{w}(G)<n-D+1$ and $c d n_{w}(G)>n-D+1$. For the graph $G$ given in Figure 3.1(a), $n=6$, $D=4, c d n_{w}(G)=5$ so that $c d n_{w}(G)>n-D+1$. For the graph $G$ given in Figure 3.1(b), $n=8, D=4$ and $c d n_{w}(G)=5$ so that $c d n_{w}(G)=n-D+1$. For the graph $G$ given in Figure 3.1(c), $n=6, D=4$ and $c d n_{w}(G)=2$ so that $c d n_{w}(G)<n-D+1$.

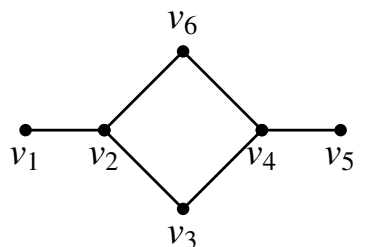

(a)

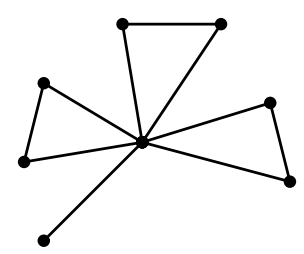

(b)

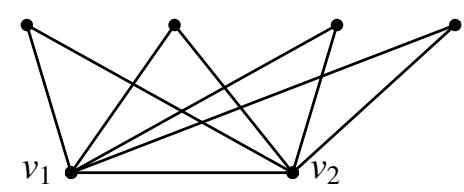

(c)

Figure 3.1: G

Theorem 3.2. Let $G$ be a connected graph of order $n \geq 2$. If $D=n-1$, then $c d n_{w}(G) \geq n-D+1$.

Proof. For any graph $G, c d n_{w}(G) \geq 2$. Since $D=n-1$, we have $n-D+1=2$ and so $c d n_{w}(G) \geq n-D+1$. 
Remark 3.3. The converse of the Theorem 3.2 is not true. For the graph $G$ given in Figure $3.1(b)$, as in the Remark 3.1, $c d n_{w}(G)=n-D+1$, but $D \neq n-1$. Also for the graph $G$ given in Figure 3.1 (a), as in the Remark 3.1, $c d n_{w}(G)>n-D+1$, but $D \neq n-1$.

Theorem 3.4. Let $R, D, k$ be three positive integers such that $k>D$ and $R<D \leq 2 R$. Then there exists a connected graph $G$ such that $\operatorname{rad}_{D} G=$ $R, \operatorname{diam}_{D} G=D$ and $c d n_{w}(G)=k$.

Proof. Case 1: When $R=1$ and $D=2$, let $G=K_{1, k-1}$. Clearly $\operatorname{rad}_{D} G=$ $1, \operatorname{diam}_{D} G=2$ and by corollary $2.15, c d n(G)=k$.

Case 2: When $R \geq 2$ and $R<D \leq 2 R$, we construct a graph $G$ with the desired properties as follows: Let $C_{R+1}: v_{0}, v_{1}, \ldots v_{R}, v_{0}$ be a cycle of order $R+1$ and let $P_{D-R+1}: u_{0}, u_{1}, \ldots, u_{D-R}$ be a path of order $D-R+1$. Let $H$ be the graph obtained from $C_{R+1}$ and $P_{D-R+1}$ by identifying $v_{0}$ of $C_{R+1}$ with $u_{0}$ of $P_{D-R+1}$. The required graph $G$ is obtained from $H$ by adding $k-D+R-2$ new vertices $w_{1}, w_{2}, \ldots, w_{k-D+R-2}$ to $H$ and joining each $w_{i}(1 \leq i \leq k-D+R-2)$ to the vertex $u_{D-R-1}$ and is shown in Figure 3.2. Clearly, $G$ is connected such that $\operatorname{rad}_{D} G=R$ and $\operatorname{diam}_{D} G=D$. Now, we show that $c d n_{w}(G)=k$. Let $S=\left\{u_{0}, u_{1}, \ldots u_{D-R-1}, u_{D-R}, w_{1}, w_{2}, \ldots w_{k-D+R-2}\right\}$ be the set of all cut-vertices and end-vertices. However, by Corollary 2.13, $S$ is not a connected weak edge detour set of $G$. Let $T=S \cup\{v\}$, where $v \in\left\{v_{R}, v_{1}\right\}$ is a vertex in $C_{R+1}$. Then $T$ is a connected weak edge detour basis of $G$ so that $c d n_{w}(G)=k$.

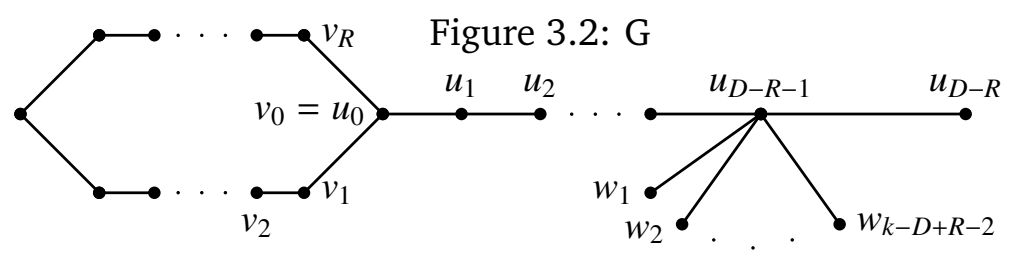

\section{References}

[1] F. Buckley and F. Harary, Distance in Graphs. Reading, MA: Addison-Wesley, 1990.

[2] G. Chartrand, H. Escuadro and P. Zhang, "Detour Distance in Graphs," J. Combin. Math. Combin. Comput., vol. 53, pp. 75-94, 2005.

[3] G. Chartrand, L. John and P. Zhang, "The Detour Number of a graph," Util. Math. vol. 64, pp. 97-113, 2003.

[4] F. Harary, Graph Theory. New Delhi: Narosa, 1997.

[5] A. P. Santhakumaran and S. Athisayanathan, "Weak edge detour number of a graph," Ars Combin., vol. 98, pp. 33-61, 2011.

[6] A. P. Santhakumaran and S. Athisayanathan, "The connected detour number of a graph,” J. Combin. Math. Combin. Comput., vol. 69, pp. 205-218, 2009. 